\title{
Excess Spin and the Dynamics of Antiferromagnetic Ferritin
}

\author{
J.G.E. Harris, J.E. Grimaldi, and D.D. Awschalom \\ Department of Physics, University of California, Santa Barbara, CA 93106 \\ A. Chiolero and D. Loss \\ Department of Physics and Astronomy, University of Basel, \\ Klingelbergstrasse 82, 4056 Basel, Switzerland
}

(October 15, 2018)

\begin{abstract}
Temperature-dependent magnetization measurements on a series of synthetic ferritin proteins containing from 100 to $3000 \mathrm{Fe}$ (III) ions are used to determine the uncompensated moment of these antiferromagnetic particles. The results are compared with recent theories of macroscopic quantum coherence which explicitly include the effect of this excess moment. The scaling of the excess moment with protein size is consistent with a simple model of finite size effects and sublattice noncompensation.
\end{abstract}

PACS numbers: 73.40.Gk, 75.60.Jp, 75.10.Jm

In nanometer-scale magnetic particles it is possible to observe a number of phenomena which do not exist in bulk samples. The evolution of magnetic order from individual atoms to large clusters, 1 thermal relaxation of the magnetization from a metastable state, 2 the different roles of surface and interior atoms, 3 and the quantum mechanical dynamics of the order parameter 4 have all received considerable experimental and theoretical attention in the last several years. Here we present measurements on a series of well-characterized samples of biomimetic antiferromagnetic particles with sizes from 100 to $3000 \mathrm{Fe}(\mathrm{III})$ ions per particle. By separating the bulk from the surface contributions to the magnetization, we explore the connection between two of these phenomena: the role of the excess moment in the macroscopic quantum coherence of antiferromagnetic particles.

Past theoretical work suggested that the Néel vector of a small antiferromagnetic particle could exhibit macroscopic quantum coherence (MQC), in which it tunnels resonantly between degenerate easy directions, at a rate accessible to experiment. 1 - Measurements of the magnetic noise spectrum and ac susceptibility of the antiferromagnetic cores of the protein ferritin revealed a resonance whose frequency scaled with particle size, applied magnetic field, temperature, and interparticle separatip in qualitative agreement with theoretical predictions. In the interpretation of these results, it was assumed that any excess moment of the ferritin cores would follow the dynamics of the Néel vector without affecting it. Measurements on natural ferritin have shown that the cores do have a small net magnetic moment $(\approx 100$ 's of $\left.\mu_{B}\right)$, 1013 presumably due to the preferential population of one magnetic sublattice during the formation of the particles. Recent theoretical work 14,5 predicts that an excess spin $\approx 100$ will have a small but appreciable effect on the MQC frequency of an antiferromagnetic particle. Motivated by this prediction and the opportunity to test long standing model 16 of the size dependence of the excess moment in small antiferromagnetic particles, we have measured the excess moments of several artificially synthesized ferritin samples.

Occurring in a wide range of plants, animals, and bacteria, ferritin consists of an organic hollow spherical shell with an inner core $\sim 80 \AA$ in diameter. This protein absorbs Fe ions through channels in its shell where they nucleateinto an insulating antiferromagnetic crystal $\left(T_{N}=\right.$ $240 K-7)$ similar to ferrihydrite. Natural ferritin can contain at most $4500 \mathrm{Fe}(\mathrm{III})$ ions, and typically contains an average of 2000. Using synthetic chemical techniques, it is possible to prepare samples in which each protein shell contains a fairly well-specified number of Fe(III) ions. The proteins used in these measurements have been extensively characterized elsewhere 10 The samples have nominal loadings of $n=100,250,500,1000,2000$, and $3000 \mathrm{Fe}(\mathrm{III})$ ions (ionic moment $\mu_{F e(I I I)}=5.92 \mu_{B}$ ) per protein. Transmission electron microscopy (TEM) measurements of the mean and variance of the core diameters have been made for all but the smallest two samples, and published elsewhere. 9 For magnetization measurements, a dilute $(\sim 0.5 \mathrm{mg} / \mathrm{ml})$ solution of a sample is dried on a polypropelene film and mounted on a twisted $\mathrm{Cu}$ wire in a commercial SQUID magnetometer. Measurements are made at temperatures ranging from $\mathrm{T}=4-300 \mathrm{~K}$ and applied fields $\mathrm{H}=0-5 \mathrm{~T}$.

Typical $\mathrm{M}(\mathrm{H})$ curves for the $n=2000$ and $n=3000$ sample are shown in Fig.1 and reveal the presence of two components: one which saturates at large $\mathrm{H}$, and a second that is approximately linear in $\mathrm{H}$. Néel 19 modeled small antiferromagnetic particles with net moments as an order parameter (essentially the Néel vector) possessing a magnetic moment $\mu$ as well as parallel and perpendicular susceptibilities $\chi_{\|}$and $\chi_{\perp}$. The energy of such a particle can be written as

$$
E=-\frac{1}{2} \chi_{\|} H^{2} \cos ^{2} \psi-\frac{1}{2} \chi_{\perp} H^{2} \sin ^{2} \psi-\mu H \cos \psi
$$

where $H$ is the applied field and $\psi$ is the angle between the Néel vector and $H$. The thermodynamic magneti- 
zation $M(H)=k_{B} T \frac{\partial}{\partial H} \ln [Z]$, where $Z$ is the partition function, can be calculated explicitly, but for $H<\mu / \chi_{\perp}$ (which holds for all measurements here), we use the approximate expression:19120

$$
M(H)=\chi_{\|} H+\left(\mu+2\left(\chi_{\perp}-\chi_{\|}\right) k_{B} T / \mu\right) L\left[\frac{\mu H}{k_{B} T}\right],
$$

where $L[\mathrm{x}]$ is the Langevin function. Because Eq.(2) assumes thermodynamic equilibrium, all of our measurements are made abgye the blocking temperature $T_{B}$ (which is size dependent 9 ) of each sample. This also justifies our ignoring anisotropy energy terms in Eq.(11).

Figs.1(a) and 1(c) show a fit to Eq.(2) for the data from the $n=2000$ and $n=3000$ samples a few Kelvin above $T_{B}$. The fit fails in the region where $M(H)$ has the most curvature, as there is a non-negligible spread in the particle sizes in each sample. This results in a departure from Eq.(2) which is difficult to model without knowing the precise size distribution in the sample. However, we can exploit the fact that the susceptibility near zero field is simply the average susceptibility of the sample. We first subtract the component of $\mathrm{M}(\mathrm{H})$ which is linear at high fields where the Langevin function has saturated to remove both the contribution from the antiferromagnetic bulk (the first term in Eq.(22)) and any diamagnetic background. The data is then normalized so that the saturation moment is unity and $\mu$ is extracted from the slope of the low field data, $\mu /\left(3 k_{B} T\right)$. This approach has the advantage of being insensitive to the total number of particles in the sample as well as to the details of its size distribution since it returns the average $\mu$ of the sample. Figs.1(b) and 1(d) show fits to the high- and low-field data from Figs.1(a) and 1(c) using Eq.(2). The fits represent the theoretical $\mathrm{M}(\mathrm{H})$ of a particle with $\mu$ equal to the average $\mu$ of the sample.

This procedure can be used at any temperature above $T_{B}$ (i.e., whenever the magnetic moments are in thermal equilibrium on the time scale of the measurement). We repeated measurements of the type shown in Fig.1 over a temperature range from roughly $T_{B}$ to $4 T_{B}$. In the samples with larger cores (Fig.2(a)), we find a weak temperature dependence of the extracted $\mu$, increasing by $\sim 20 \%$ over a factor of 4 in temperature. For the smallest cores (Fig.2(b)), the temperature dependence of $\mu$ is quite strong, increasing by roughly a factor of two over the same range. A similar trend has been observed in natural ferritin as well as $\mathrm{NiO}$ particles.22 13 The reason for this temperature dependence is not clear. It should be noted that the model behind Eqs.(11) and (2) does not take into account any of the microscopic phenomena which might alter the properties of a small antiferromagnetic particle except in as much as these effects can be modeled by $\mu, \chi_{\|}$and $\chi_{\perp}$. Weaker exchange 16 strong radial anisotropies, 21 and frustration 22 can exist at the surfaces of such particles, and may be responsible for the observed temperature dependence of $\mu$. Multiple sublattices can also exist in very small particles of a material which is antiferromagnetic in the bulk. $\mathrm{B}$ At the low concentrations used here the typical interparticle dipolar fields should be well below $1 \mathrm{G}$, too small to account for these effects. For comparison with MQC, which is only observed below $200 \mathrm{mK}$, we extrapolate the linear temperature dependence shown in Fig. 2 to $\mathrm{T}=0$ in order to extract the relevant excess moment.

The result of this extrapolation is shown in Fig.3, where $\mu$ is plotted vs. $n$. The vertical error bars represent the combined effects of the reproducibility between identically prepared samples and the uncertainties in the linear extrapolations of Fig.2, $10 \%$. Because the extracted $\mu$ corresponds to the excess moment averaged over the sample, the horizontal error bars do not represent the variance in particle size, but rather the uncertainty in the mean particle size, estimated from the discrepancies between the nominal loading and the particle size measured by TEM $(\sim 20 \%)$. This is probably an underestimate in the case of the smallest two samples. The data are fit by a $0.56 \pm 0.05$ power law. If one plots $\mu$ in units of $\mu_{F e(I I I)}$, the coefficient of the power law fit is 1.15 , quite close to unity. Néel has suggested three models of imperfect sublattice compensation in small antiferromagnetic particles. 16 In the first, an antiferromagnetic particle has a surface consisting of sites belonging to one sublattice only, giving $\mu=c n^{\frac{2}{3}} \mu_{F e(I I I)}$. The proportionality constant $c$ is roughly 4 for the platonic solids. In the second model, the surface sites are distributed randomly between the two sublattices; then one has a random walk over the surface, and so $\mu=c^{\frac{1}{2}} n^{\frac{1}{3}} \mu_{F e(I I I)}$. For a particle surface of fractal dimension (as predicted by models in which the ferritin core is formed by diffusion limited aggregation 23), the random walk over the surface can give any power law from $1 / 3$ to $1 / 2$. Lastly, if all the ions (as opposed to merely the surface ones) are randomly distributed between the sublattices, then $\mu=n^{\frac{1}{2}} \mu_{F e(I I I)}$. This can occur, for example, if there is a non-stochiometric replacement of some magnetic ions with non magnetic ions. This prediction (which has no free parameters) is plotted in Fig.3 as a dashed line. The agreement between this last prediction and the data might be strong evidence for the random population of the sublattices throughout the volume of the particles. We note, however, that for particles with 100 to 3000 magnetic ions the discreteness of the lattice, combined with any surface roughness, means that a disproportionately large number of sites will be located on the surface. Thus, it is not possible to determine whether the sublattice non compensation is a volume effect or a surface effect. That the power law is clearly much less than 1 is strong evidence that the excess moment does not result from canting of the sublattices. Measurements made on fully loaded natural ferritin $(\mathrm{n}=4500)$ and partially loaded natural ferritin $\left(n_{-2} 2000\right)$ are in good agreement with a $1 / 2$ power law. 113

We can now compare the measured values of the excess spin and the MQC resonance frequency $\nu_{M Q C}$ as a functions of $n$. An antiferromagnet strongly coupled to an uncompensated moment can be described by the 
effective action 14.15

$$
\begin{aligned}
S_{E}=V \int d & \left\{\frac{\chi_{\perp}}{2 \gamma^{2}}\left(\dot{\theta}^{2}+\dot{\phi}^{2} \sin ^{2} \theta\right)+K_{y} \sin ^{2} \theta \sin ^{2} \phi\right. \\
+ & \left.K_{z} \cos ^{2} \theta\right\}+i \hbar S \int d \tau \dot{\phi}(1-\cos \theta),
\end{aligned}
$$

where $\theta$ and $\phi$ are the spherical coordinates of the Néel vector, $V$ is the volume of the grain, $K_{z} \geq K_{y}>0$ its magnetic anisotropies, $\gamma=2 \mu_{B} / \hbar$, and $S$ is the magnitude of the excess spin. Let us define $S_{\mathrm{AFM}}=$ $\hbar V \sqrt{2 K_{y} \chi_{\perp}} / \mu_{B}$, the instanton action one would obtain for an antiferromagnet without an uncompensated moment.

We will use instanton techniques to calculate the tunnel splitting. It has been shown 15 that in the regime $K_{y} \ll K_{z}$ instanton solutions have an approximate frequency

$$
\omega_{\mathrm{Ferri}}=\frac{2 \lambda V}{\hbar S} \sqrt{K_{y} K_{z}},
$$

with

$$
\lambda=\left(1+\frac{1}{4} \frac{K_{z}}{K_{y}}\left(\frac{S_{\mathrm{AFM}}}{\hbar S}\right)^{2}\right)^{-1 / 2} .
$$

and an action

$$
\begin{aligned}
& S_{\mathrm{Ferri}}=\frac{2 \hbar S}{\lambda} \sqrt{K_{y} / K_{z}}\left\{1+\frac{1}{3} \lambda^{2} \frac{K_{y}}{K_{z}}+\right. \\
&\left.\frac{1}{8} \lambda^{4}\left(\frac{S_{\mathrm{AFM}}}{\hbar S}\right)^{2}\left(1+\delta\left(\sqrt{\frac{K_{y}}{K_{z}}} \lambda\right)\right)\right\} .
\end{aligned}
$$

where

$$
\delta(x)=\frac{1}{x^{3}}\left(\sqrt{1+x^{2}} \operatorname{arcsinh}(x)-x-x^{3} / 3\right) .
$$

The tunnel splitting is then given by

$$
\Delta_{0}=8 \hbar \omega_{\text {Ferri }} \sqrt{\frac{S_{\text {Ferri }}}{2 \pi \hbar}}|\cos (\pi S)| e^{-S_{\text {Ferri }} / \hbar},
$$

and the crossover temperature to the quantum regime by

$$
k_{B} T^{*}=K_{y} V \hbar / S_{\mathrm{Ferri}} .
$$

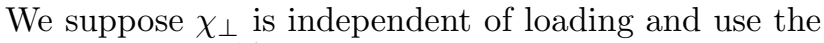
simple estimate $\chi_{\perp}=\mu_{B}^{2} N / k_{B} T_{N} V$. The volume of the magnetic core of a fully loaded grain is roughly that of a sphere with a diameter of $7.5 \mathrm{~nm}$, and contains 4500 ions. $T_{N}=240 \mathrm{~K}$ in ferritin, which gives $\chi_{\perp} \approx$ $5 \cdot 10^{-5} \mathrm{emu} / \mathrm{G} \mathrm{cm}^{3}$.

The crossover temperature to the quantum regime has only been measured in fully loaded grains, where $T^{*}=$ $200 \mathrm{mK}$. In the absence of experimental data we will assume $T^{*}$ is also independent of loading.

Armed with these values for $\chi_{\perp}$ and $T^{*}$, we are left with two unknowns at each loading, the anisotropies $K_{y}$ and $K_{z}$. We can deduce their values from Eqs. (8) and (9), using the experimental values for $\mu$ and $\nu_{M Q C}$ listed in Table I. We see that the hypothesis that $K_{y} \ll K_{z}$ is at least self-consistent, and that the anisotropies are not strongly size dependent. The only exception is the value obtained for $K_{z}$ in fully loaded grains. We note that the measurement of $\mu$ in fully loaded natural ferritin 23 lies somewhat below the power law shown in Fig.3, possibly as the result of slightly different sample preparation, interparticle effects (the samples were not diluted), or differences in the surface of a grain completely filling the spherical protein shell. The value of $K_{y}$ increases somewhat for smaller particles, consistent with the trend in blocking temperature as a function of $n, 18$ though it is somewhat smaller than typical for antiferromagnetic particles. We are not aware of any other measurements of the transverse anisotropy $K_{z}$ in such systems.

In conclusion, we have measured the excess moment of the antiferromagnetic protein ferritin as a function of the number of magnetic ions per protein. Using diluted samples to ensure the absence of interparticle interactions, we find an approximately square root dependence of the excess moment upon particle size, in agreement with a simple model (which has no free parameters) of imperfect sublattice compensation. We use this result to compare recent theoretical work on the effect of an excess moment to earlier MQC and blocking temperature measurements in the same samples.

We are grateful to Steven Mann and Trevor Douglas formerly of the University of Bath, for providing the artificial ferritin samples. This work was supported by the AFOSR \# F49620-99-1-0033.

${ }^{1}$ Jing Shi, S. Gider, K. Babcock, D.D. Awschalom, Science 271, 937 (1996).

${ }^{2}$ W. Wernsdorfer, E.B. Orozco, K. Hasselbach, A. Benoit, B. Barbara, N. Demoncy, A. Loiseau, H. Pascard, and D. Mailly, Phys. Rev. Lett. 78, 1791 (1997).

${ }^{3}$ R.H. Kodama, Salah A. Makhlouf, and A.E. Berkowitz, Phys. Rev. Lett. 79, 1393 (1997).

${ }^{4}$ E.M. Chudnovsky and L. Gunther, Phys. Rev. Lett. 60, 661 (1988); G. Scharf, W.F. Wreszinski, and J.L. Hemmen, J. Phys. A 20, 4309 (1987).

${ }^{5}$ B. Barbara, E.M. Chudnovsky, Phys. Lett. A 145, 205 (1990).

${ }^{6}$ I.V. Krive, O. B. Zaslawski, J. Phys.: Condens. Matter 2, 9457 (1990).

${ }^{7}$ D.D. Awschalom, J.F. Smyth, G. Grinstein, D.P. DiVincenzo, D. Loss, Phys. Rev. Lett. 68, 3092 (1992).

${ }^{8}$ S. Gider, D.D. Awschalom, in Quantum Tunneling of Magnetization, edited by L. Gunther and B. Barbara (Kluwer, 1994), p. 243.

${ }^{9}$ S. Gider, D.D. Awschalom, T. Douglas, S. Mann, M. Cha- 


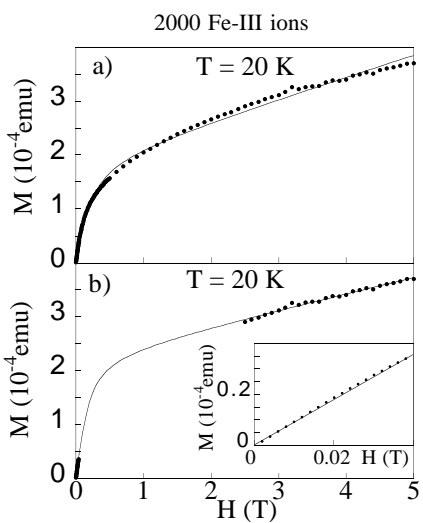

FIG. 1. Magnetization vs. applied field above the blocking temperature for samples with particle size $n=2000 \mathrm{Fe}$ (III) ions (a,b) and $n=3000(\mathrm{c}, \mathrm{d})$. The solid line is a fit to the form of Eq.(2), where in (b) and (d), only the low- and high-field data are fit, as described in the text. The insets in (b) and (d) are magnifications of the low-field data and fit.

parala, Science 268, 77 (1995).

10 Alain Blaise, Jacques Feŕon, Jean-Luc Girardet, JeanJacques Lawrence, C. R. Acad. Sci. 265, 1077 (1967).

${ }^{11}$ M-E.Y. Mohie-Eldin, R.B. Frankel, L. Gunther, J. Magn. Magn. Mater. 135, 65 (1994).

12 S. H. Kilcoyne, R. Cywinski, J. Magn. Magn. Mater. 140144, 1466 (1995).

${ }^{13}$ Salah A. Makhlouf, F.T. Parker, and A.E. Berkowitz, Phys. Rev. B. 55, 14717 (1997).

14 D. Loss, D.D. DiVincenzo, G. Grinstein, Phys. Rev. Lett. 69, 3232 (1992).

${ }^{15}$ A. Chiolero, D. Loss, Phys. Rev. B 56, 738-746 (1997).

${ }^{16}$ Louis Néel, C. R. Acad. Sci. 252, 4075 (1961).

${ }^{17}$ E.R. Bauminger, I. Nowik, Hyp. Int. 50, 489 (1989).

18 S. Gider, D.D. Awschalom, T. Douglas, K. Wong, S. Mann, G. Cain, J. Appl. Phys. 79, 5324 (1996)

${ }^{19}$ Louis Néel, C. R. Acad. Sci. 253, 9 (1961).

${ }^{20}$ Savas Gider, Ph.D Dissertation, University of California at Santa Barbara, 1996

${ }^{21}$ I.S. Jacobs and C.P. Bean in Magnetism, edited by G.T. Rado and H. Suhl (Academic Press, New York, 1963), Vol. III, p.314.

${ }^{22}$ R.H. Kodama, A.E. Berkowitz, E.J. McNiff, Jr., and S. Foner, Phys. Rev. Lett. 77, 394 (1996).

${ }^{23}$ R. B. Frankel, G. C. Papaefthymiou, G. D. Watt, Hyperfine Interactions 66, 71 (1991)

${ }^{24}$ V.G. Bar'yakhtar, B.A. Ivanov, M.V. Chetkin, Sov. Phys. Usp. 28, 563 (1985).

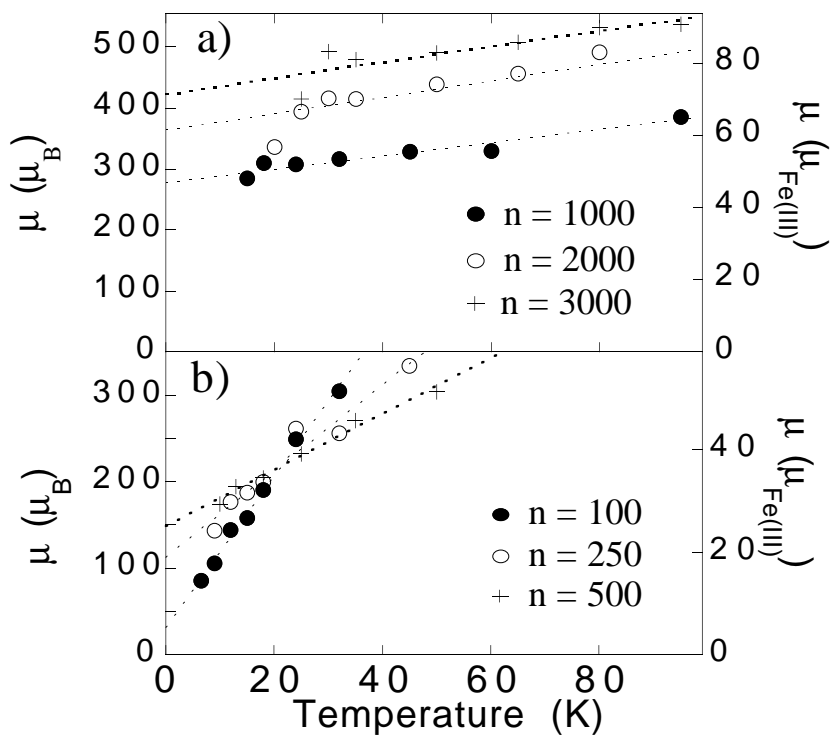

FIG. 2. The excess moment $\mu$ (in units of $\mu_{B}$ and the ionic moment $\left.\mu_{F e(I I I)}\right)$ of ferritin cores each with $n \mathrm{Fe}(\mathrm{III})$ ions as a function of temperature from $T_{B}$ to roughly $4 T_{B}$ for each sample. The dashed lines are linear fits to, in (a) the larger cores, and in (b) the smaller cores. The temperature scale is the same in both a) and b).

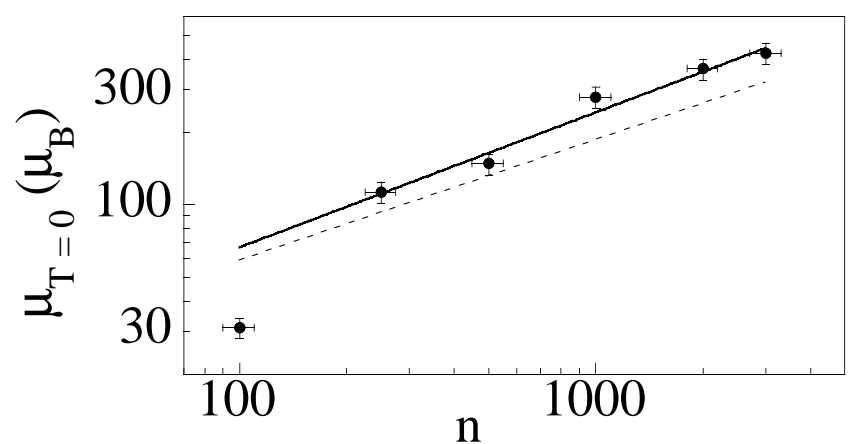

FIG. 3. The $\mathrm{T}=0$ excess moment in units of $\mu_{B}$ as a function of particle size $\mathrm{n}$. The solid line is a power law fit giving an exponent of 0.56 . The dashed line is the no free parameter prediction of the $\mu=n^{\frac{1}{2}} \mu_{F e(I I I)}$ model described in the text.

\begin{tabular}{lllll}
\hline \hline$n$ & $\begin{array}{l}\mu \\
{\left[\mu_{B}\right]}\end{array}$ & $\begin{array}{l}\nu_{M Q C} \\
{[\mathrm{~Hz}]}\end{array}$ & $\begin{array}{l}K_{y} \\
{\left[\mathrm{erg} / \mathrm{cm}^{3}\right]}\end{array}$ & $\begin{array}{l}K_{z} \\
{\left[\mathrm{erg} / \mathrm{cm}^{3}\right]}\end{array}$ \\
\hline 100 & 31 & & & \\
250 & 113 & & & \\
500 & 148 & & $3.8 \cdot 10^{3}$ & $6.1 \cdot 10^{6}$ \\
1000 & 278 & $1.6 \cdot 10^{8 *}$ & $2.1 \cdot 10^{3}$ & $7.7 \cdot 10^{6}$ \\
2000 & 401 & $7.8 \cdot 10^{7 *}$ & $1.9 \cdot 10^{3}$ & $4.5 \cdot 10^{6}$ \\
3000 & 423 & $5.6 \cdot 10^{6 *}$ & $1.5 \cdot 10^{3}$ & $1.9 \cdot 10^{6}$ \\
4500 & $316^{\natural}$ & $9.40 \cdot 10^{5 \sharp}$ & $1.9]$ \\
${ }^{*}$ MQC measurements in artificial ferritin, ref. 9$]$ \\
\# MQC measurements in natural ferritin, ref. 6 \\
घ Ref. 12 \\
12
\end{tabular}

TABLE I. Excess moment $\mu$, MQC resonance frequency $\nu_{M Q C}$, and anisotropy energies $K_{y}$ and $K_{z}$ for ferritin cores containing $n \mathrm{Fe}(\mathrm{III})$ ions. 